\title{
Editorial
}

\section{Impact of Kirk's Results on the Development of Fixed Point Theory}

\author{
Mohamed A. Khamsi ${ }^{1,2}$ and Tomas Dominguez-Benavides ${ }^{3}$ \\ ${ }^{1}$ Department of Mathematical Sciences, University of Texas at El Paso, El Paso, TX 79968, USA \\ ${ }^{2}$ Department of Mathematics and Statistics, King Fahd University of Petroleum E Minerals, P.O. Box 411, \\ Dhahran 31261, Saudi Arabia \\ ${ }^{3}$ Departamento de Análisis Matemático, Universidad de Sevilla, 41080 Sevilla, Spain
}

Correspondence should be addressed to Mohamed A. Khamsi, mohamed@utep.edu

Received 31 December 2010; Accepted 31 December 2010

Copyright (C 2010 M. A. Khamsi and T. Dominguez-Benavides. This is an open access article distributed under the Creative Commons Attribution License, which permits unrestricted use, distribution, and reproduction in any medium, provided the original work is properly cited.

"The theory of Fixed Points is one of the most powerful tools of modern mathematics" is a quote by Felix Browder, who in the mid-nineteen sixties gave a new impetus to the modern fixed point theory via the development of nonlinear functional analysis as an active and vital branch of mathematics. The metric fixed point theory is a rather loose knit branch of fixed point theory concerning methods and results that involve properties of an essentially isometric nature. The divide between the metric fixed point theory and the more general topological theory is often a vague one. The use of successive approximations to establish the existence and uniqueness of solutions is at the origin of the metric theory. It goes back to Cauchy, Liouville, Lipschitz, Peano, Fredholm, and especially Picard. However, it is the Polish mathematician S. Banach who is credited with placing the underlying ideas into an abstract framework suitable for broad applications well beyond the scope of elementary differential and integral equations.

Kirk's fixed point theorem published in 1965 had a profound impact on the development of the fixed point theory over the last 40 years. Through the concept of generalized distance, Tarski's classical fixed point theorem may be seen as a variant of Kirk's fixed point theorem in discrete sets. This shows among other things the power of this theorem.

This special issue focused on many types of applications of Kirk's fixed point theorem. It included nonexpansive mappings in Banach and metric spaces, multivalued mappings in Banach and metric spaces, monotone mappings in ordered sets, multivalued mappings in ordered sets, applications to nonmetric spaces like modular function spaces, and applications to logic programming and directed graphs. 
The special issue contains 38 papers accepted. Most of the papers touched on all applications of Kirk's fixed point theorem.

Mohamed A. Khamsi

Tomas Dominguez-Benavides 